\title{
Pilimiction, a Rare Presentation of Ovarian Teratoma: A Case Report
}

\author{
Feysel Hassen Issack (1D', Seid Mohammed Hassen (D)', Ferid Ousman Mummed (D)', \\ Kaleab Habtemichael Gebreselassie (D)', Seid Kedir Hassen², Ibsa Kedir Hassen (iD) \\ 'Department of Surgery, Urology Unit, St. Paul's Hospital Millennium Medical College, Addis Ababa, Ethiopia; ${ }^{2}$ Department of Obstetrics and \\ Gynecology, St. Paul's Hospital Millennium Medical College, Addis Ababa, Ethiopia \\ Correspondence: Feysel Hassen Issack, Department of Surgery, Urology Unit, St. Paul's Hospital Millennium Medical College, Addis Ababa, Ethiopia, \\ Tel+2519325III45, Email feyselh@gmail.com
}

\begin{abstract}
Background: Adnexal teratoma involving the urinary bladder is a very rare condition. Presentation is variable ranging from irritative LUTS (lower urinary tract symptoms) to pilimiction or trichiuria (passage of hair in the urine).

Case Presentation: We report a case of a 42-year-old woman who presented with pilimiction and lower abdominal pain. Contrastenhanced computed tomography scan (CECT) and cystoscopy were used for the diagnosis. Tumor markers were negative. Right side salpingo-oophorectomy and partial bladder wall excision were performed. Histopathology of the specimen showed features consistent with mature teratoma. The patient reported improvement of symptoms in the subsequent follow-up visits.

Conclusion: Pilimiction is a pathognomonic sign of bladder teratomas. This case represents a rare complication of ovarian teratoma. Therefore, a high index of suspicion is required in patients with ovarian teratoma presenting with urinary complaints. Transurethral resection is associated with a high recurrence rate and is not recommended for secondary bladder teratomas.
\end{abstract}

Keywords: pilimiction, adnexal mass, bladder teratoma, trichiuria, case report

\section{Introduction}

Teratomas are tumors consisting of three germ layers seen commonly during childhood. Mature teratomas are benign and demonstrate well-differentiated tissues, such as sebaceous glands, hair, and teeth. Adnexal teratoma involving the urinary bladder is a very rare condition. The clinical presentation may vary from irritative lower urinary tract symptoms (LUTS) or urinary retention to pilimiction (passage of hair in the urine). We hereby present a case of mature teratoma of the ovary involving the urinary bladder, which primarily manifested with pilimiction. The condition is rare, and there are only a few similar reports in the literature.

\section{Case Presentation}

A 42-year-old Para I female patient was referred to a tertiary hospital after she presented with a passage of hair in the urine and lower abdominal pain for a year. Otherwise, there was no complaint of urinary frequency, urgency, or pain on urination. She did not report fever, foul-smelling vaginal discharge, or pain during coitus. She has not had any known chronic medical illness.

On physical examination, the patient had stable vital signs. There was no remarkable positive physical finding other than an old Pfannenstiel surgical scar. The pelvic exam was negative for any visible genital lesions, cervical motion tenderness, or palpable adnexal mass.

She underwent a joint evaluation and workup by urology and gynecologic oncology units. On laboratory investigation, $\mathrm{CBC}$ (complete blood count) was normal and hematocrit was $40.3 \%$. Urine analysis was remarkable for microscopic hematuria in the range of 3-5 RBC/HPF (Normal $\leq 2 \mathrm{RBCs} / \mathrm{HPF}$ ). But the other parameters of urine analysis were in the 

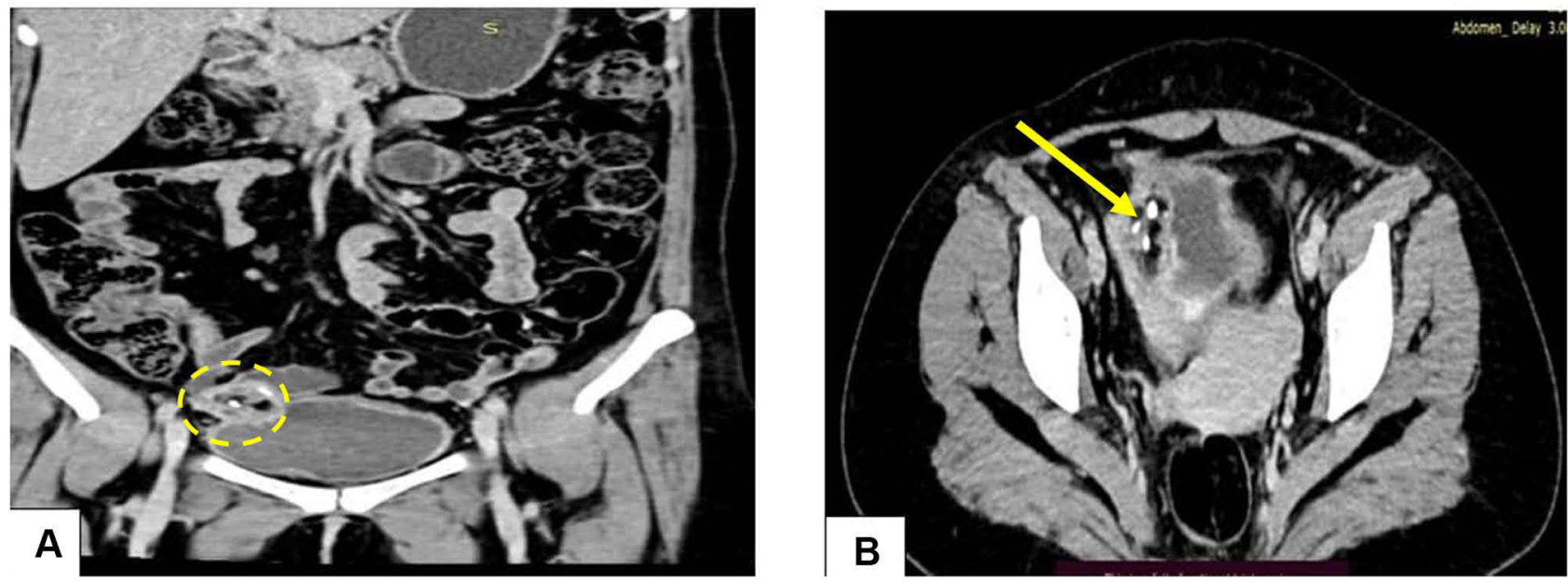

Figure I CECT of the abdomen and pelvis. Right adnexal mass with bladder involvement. (A) Coronal reformatted (dotted circle). (B) Axial image (arrow).

normal range. The serum creatinine, blood urea nitrogen (BUN), serum electrolytes, and liver enzymes were also normal. Serologic tests for HIV, hepatitis B, and C as well as Treponema were negative.

Abdominopelvic ultrasound examination showed a bizarre shaped tubular echogenic shadow in the lumen of the bladder that looks like a foreign body. Further imaging with a contrast-enhanced CT scan of the abdomen and pelvis showed a complex heterogeneous mass lesion in the right adnexa measuring $5 \mathrm{~cm} * 3.7 \mathrm{~cm}$ that has multiple calcifications and fat attenuations inside it. The lesion invaded the right superolateral surface of the bladder with a defect in the bladder mucosa. It was concluded as a right adnexal mass lesion with possible bladder invasion (Figure 1).

Cystoscopy was also done and revealed a whitish papillary mass with hair-like component over the right posterior wall of the bladder near the dome (Figure 2).

Further workup with tumor markers using serum beta HCG, serum LDH, CA-125, and CEA did not reveal any clue toward a specific diagnosis.

A joint decision was made to surgically explore the patient. The pelvis was entered through the previous Pfannenstiel incision. The finding was a $4 \mathrm{~cm} * 6 \mathrm{~cm}$ right ovarian mass with solid and cystic areas containing hair, bone, and teeth. Right side salpingo-oophorectomy, partial cystectomy and bladder repair were done. The excised mass was sent for histopathological analysis.

Excisional biopsy report showed stratified squamous epithelium, adnexal structures, fatty and bony tissue fragments, confirming the diagnosis of mature ovarian teratoma (Figure 3).

On subsequent follow-up visits, the patient reported improvement in symptoms and physical examination was unremarkable.
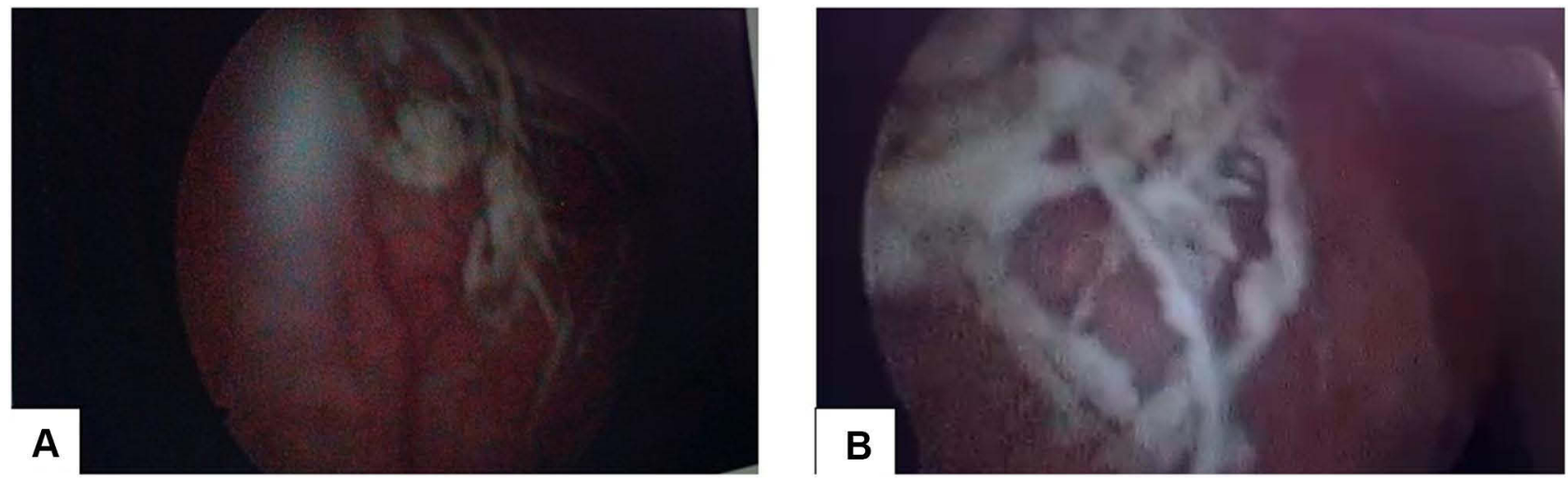

Figure 2 Cystoscopy shows hair-like structures arising from the base and posterior bladder wall. (A) Far view. (B) Near view. 

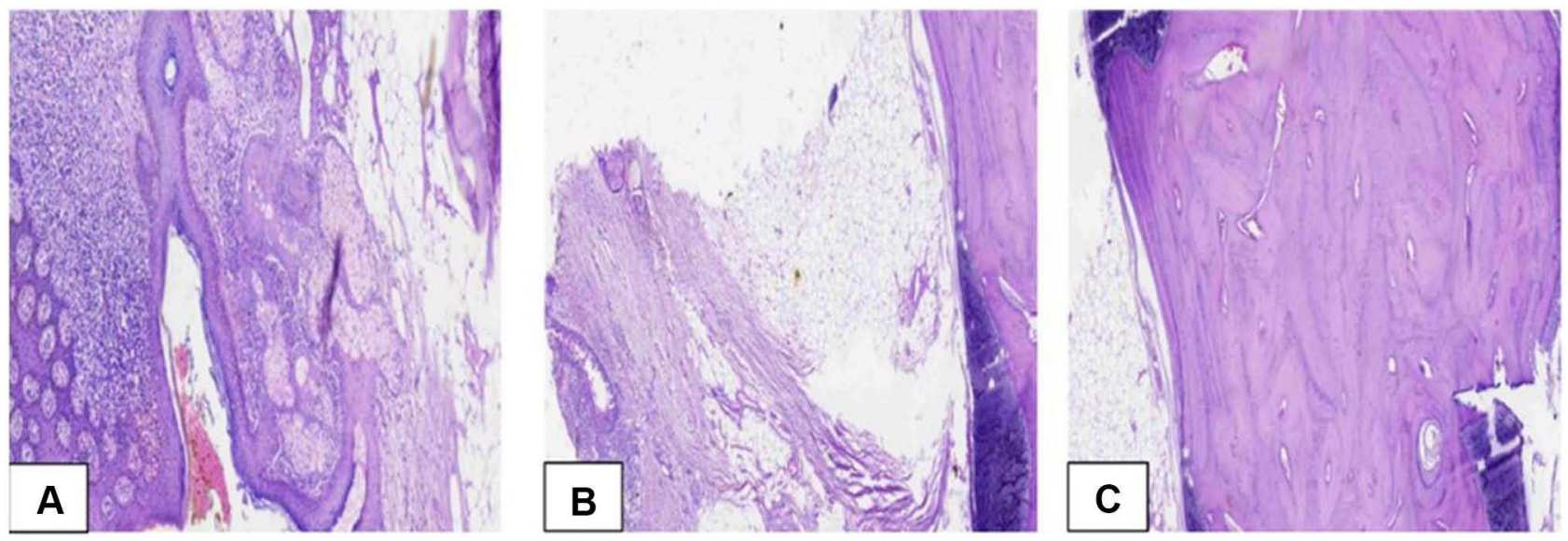

Figure 3 Scanned pictures of the histopathology specimen $(\mathbf{A}-\mathbf{C})$.

\section{Discussion}

Mature teratomas are the commonest benign tumors of the ovary. They account for $20-50 \%$ of all ovarian tumors and are more prevalent in premenopausal females. In clinical practice, they are characterized by a unilateral involvement, which is often on the right side, although up to $10 \%$ of cases can be bilateral. ${ }^{1,2}$ Pathologically, ovarian teratomas are germ cell tumors. The word was first used by Virchow in 1863 and was derived from the Greek "teras", meaning "monster". ${ }^{-4}$

Mature ovarian teratomas are indolent and asymptomatic tumors. Their diagnosis is often incidental either during a routine pelvic examination or abdominopelvic imaging performed for other indications. However, some patients may present with symptoms secondary to tumor-related complications, such as acute-abdomen, abdominal lump, LUTS, or sepsis. These are caused by torsion, tumor rupture or infection. ${ }^{4-6}$

Torsion is the commonest complication of mature ovarian teratoma occurring in $16 \%$ of the cases. Other uncommon complications include tumor rupture (1-4\%), malignant transformation (1-2\%), infection (1\%), invasion into adjacent viscera $(<1 \%)$, and very rarely, autoimmune hemolytic anemia and paraneoplastic syndrome. ${ }^{4-7}$ Invasion and rupture of the tumor might involve adjacent pelvic and abdominal structures, most commonly the urinary bladder. There are also case reports of involvement of the rectum, vagina, small intestine, sigmoid colon, anterior abdominal wall, and peritoneal cavity. ${ }^{8}$

The anatomical proximity of the urinary bladder to the ovaries makes it vulnerable to direct involvement by tumors of ovarian origin. The clinical presentation of this rare occurrence depends on the extent of bladder involvement and the biological nature of the tumor. According to our literature review, superficial involvement of the bladder wall often presents with irritative LUTS, such as frequency and urgency. On the other hand, deeper invasion into the bladder lumen by the teratoma manifests itself with urinary tract infection (UTI), hematuria, and LUTS. Many of these features are nonspecific and can be easily overlooked. Pilimiction, however, is a rare but pathognomonic feature of full-thickness bladder wall invasion by ovarian teratoma. Though the exact pathogenesis is not well understood, the leakage of teratoma contents such as hair into the urinary bladder is thought to be caused by trauma, infection, torsion, friction or pressure necrosis, and fistulation. ${ }^{3,4}$ At times, the hair in the bladder lumen might create an obstructive ball at the bladder outlet and manifest as acute urinary retention. ${ }^{1,5,6,9}$

Pilimiction was first reported in 1700 by Wallace. Its presence is a specific and diagnostic indicator of ovarian teratoma and fistula formation. Localized ovarian teratomas do not pose a diagnostic difficulty of their own. However, involvement in the urinary bladder is often diagnosed late unless patients present with pilimiction like the case in our patient.

In most similar cases reported so far, the definitive diagnosis was made through the use of cystoscopy, computed tomography (CT) scan, or laparotomy. ${ }^{6,9,10}$ There are also few reports on laparoscopic diagnosis and management of mature teratoma with bladder involvement. ${ }^{10}$ 
Previous reports attributed the pathogenesis of bladder involvement to malignant transformation of the teratoma at some point in time leading to aggressive invasion of adjacent pelvic organs. However, with detailed research of the cases and pathology specimens, it was shown that benign teratomas can also cause fistula formation with nearby structures. Intermittent leakage of tumor contents can lead to the chronic inflammatory process and adhesion formation resulting in fistulation. This is particularly common during tumor necrosis, torsion, and infection. Chronic pressure of the tumor on adjacent organs is also suspected as a possible mechanism for fistula formation. ${ }^{1,10}$

A malignant transformation should be ruled out with a histopathological examination of the surgical specimen.

\section{Conclusion}

This case represents a rare complication of ovarian teratoma. Though very rare as a presenting symptom, pilimiction is a pathognomonic sign of primary or secondary bladder teratomas. Therefore, a high index of suspicion is required in patients with ovarian teratoma presenting with urinary complaints.

In addition to the clinical history, cystoscopy and cross-sectional imaging aid in the diagnosis of bladder teratoma. Definitive diagnosis is provided by histopathology of the surgical specimen.

\section{Abbreviations}

BUN, blood urea nitrogen; CA-125, carbohydrate antigen-125; CBC, complete blood count; CEA, carcinoembryonic antigen; CECT, contrast-enhanced computed tomography; CT, computed tomography; HCG, human chorionic gonadotropin; HPF, high power field; LDH, lactate dehydrogenase; LUTS, lower urinary tract symptoms; RBC, red blood cells; UTI, urinary tract infection.

\section{Data Sharing Statement}

All the generated data are included in this article.

\section{Ethics Approval and Consent to Participate}

No institutional review board approval was required.

\section{Consent for Publication}

Written informed consent was obtained from the patient for the publication of this case report and accompanying images. A copy of the written consent is available for review by the Editor-in-Chief of this journal on request.

\section{Funding}

The authors received no funding for this work.

\section{Disclosure}

The authors have no competing interests in this work.

\section{References}

1. Hamza M, Yasmeen T, Nadeem IA, Fatima N, Fatima S, Huzaifa M. Ovarian dermoid cyst presenting with unusual complaint of hair coming out of the anal orifice-A case report. JPMA. 2020;2019. doi:10.5455/JPMA.13778

2. Kizaki Y, Nagai T, Ohara K, et al. Ovarian mature cystic teratoma with fistula formation into the rectum: a case report. SpringerPlus. 2016;5(1):1-6. doi:10.1186/s40064-016-3426-4

3. Nagamani T, Vani I, Nimmana SP, Kumar YM. Ovarian cystovesical fistula causing pilimiction: an unusual complication of ovarian cyst. Ann Women Child Health. 2015;1(1):C1-4.

4. Tandon A, Gulleria K, Gupta S, Goel S, Bhargava SK, Vaid NB. Mature ovarian dermoid cyst invading the urinary bladder. Ultrasound Obstet Gynecol. 2010;35(6):751-753. doi:10.1002/uog.7524

5. Sardesai S, Raghoji V, Dabade R, Shaikh H. Benign cystic teratoma of ovary perforating into the urinary bladder: a rare case. J Obstet Gynaecol India. 2012;1(62):54-55. doi:10.1007/s13224-013-0354-x

6. Bhasin SK, Malik SM, Sharma G, Gupta SK. Ovarian dermoid presenting as acute intestinal obstruction: a rare case report and review of literature. Int Surg J. 2016;2(2):283-285. doi:10.5455/2349-2902.isj20150533 
7. Guo H, Yin K, Wang Y, et al. Mature cystic ovarian teratoma invading the bladder: a rare case report. Transl Surg. 2018;3(3):62. doi:10.4103/ts. ts $10 \quad 18$

8. Naqvi KZ, Abdullah A, Jabeen M, Iqbal F, Edhi M. Ovarian dermoid causing pilimiction. J Coll Physicians Surg Pak. 2015;25(1):71-72. doi:10.2015/JCPSP.7172

9. Godara R, Karwasra RK, Garg P, Sharma NP. A diagnostic symptom of ovarian dermoid cyst. Internet J Gynecol Obstet. 2006 ;6(1):62-69.

10. Vaishnav A, Sarkar D, Pal DK. Bladder teratoma with pilimiction in a male adolescent. Urol Ann. 2020;12(3):286. doi:10.4103/UA.UA_125_19

Research and Reports in Urology

\section{Publish your work in this journal}

Research and Reports in Urology is an international, peer-reviewed, open access journal publishing original research, reports, editorials, reviews and commentaries on all aspects of adult and pediatric urology in the clinic and laboratory including the following topics: Pathology, pathophysiology of urological disease; Investigation and treatment of urological disease; Pharmacology of drugs used for the treatment of urological disease. The manuscript management system is completely online and includes a very quick and fair peer-review system, which is all easy to use. Visit http://www.dovepress.com/testimonials.php to read real quotes from published authors.

Submit your manuscript here: https://www.dovepress.com/research-and-reports-in-urology-journal 